\title{
Health behaviors and quality of life in Chinese survivors of cervical cancer: a retrospective study
}

This article was published in the following Dove Press journal:

OncoTargets and Therapy

23 April 2014

Number of times this article has been viewed

\section{Shu-xia Nie \\ Chuan-qiang Gao}

Department of Gynecology and Obstetrics, People's Hospital of Xintai City, Shandong Province, Affiliated to Taishan Medical University, Xintai, People's Republic of China
Correspondence: Chuan-qiang Gao Department of Thoracic Surgery, People's Hospital of Xintai City, Shandong Province, Affiliated to Taishan Medical University, Xintai 27।200,

People's Republic of China Email zlflk_2012@I63.com
Background: The aim of this study was to evaluate health behaviors and quality of life (QoL) in cervical cancer survivors, and to identify factors that may compromise or enhance their health-related QoL.

Methods: Data were collected retrospectively from the records of 102 consecutive patients with cervical cancer treated from May 2007 to January 2009 at the People's Hospital of Xintai City in Shandong Province. The study methodology was guided by the contextual model of health-related QoL.

Results: The results showed the significant mediating effects of general health status and psychological well-being between life burden and health-related QoL, between sexual impact of the disease and health-related QoL, and between the patient-doctor relationship and healthrelated QoL. In addition, there were a significant association between health-related QoL, education level, tumor stage, marital status, and age. Life burden and the patient-doctor relationship was also related to the sexual impact of the disease. However, no significant difference in health-related QoL and sexual impact was observed according to type of treatment received.

Conclusion: These results advance our understanding of the predictors of health-related QoL and the relationship between them. Health-related QoL in cervical cancer survivors may be improved by mediating life burden, sexual functioning, and the patient-doctor relationship.

Keywords: health-related quality of life, life burden, sexual functioning, patient-doctor relationship, cervical cancer

\section{Introduction}

Cervical cancer remains a health challenge nationally and globally. In the USA, more than 12,000 women are diagnosed each year with invasive cervical cancer and more than 220,000 are living with this diagnosis. ${ }^{1}$ With improved screening efforts and better treatment regimens, longer disease-free intervals and better survival rates are possible compared with a decade ago. ${ }^{2-5}$ Therefore, there is more opportunity and a greater need to examine long-term health-related quality of life (QoL) and survivorship characteristics in this population of women.

A substantial body of literature supports the need to examine health-related QoL in cervical cancer survivors. Prevalence studies have estimated that a new diagnosis of gynecological cancer is associated with moderate/severe anxiety or depression in about $50 \%$ of women. ${ }^{6}$ Increasing attention has been focused on the issues of emotional distress and QoL in women with gynecological cancer. ${ }^{7,8}$ Numerous short-term physical, psychological, social, and sexual disruptions have been noted; however, few studies have investigated the long-term impact of cervical cancer on health-related QoL. ${ }^{9-12}$ 
Long-term health-related QoL can be affected in several ways in this vulnerable group, ie, anatomical changes resulting from the cancer or its treatment may permanently impair sexual function and reproductive ability, and significantly impact self-image and social well-being. ${ }^{13-15}$ Therefore, we performed a retrospective study to evaluate health behaviors and QoL in cervical cancer survivors and to identify factors that may compromise or enhance health-related QoL.

\section{Patients and methods}

\section{Patients}

The present study was approved by the ethics committee of the People's Hospital of Xintai City in Shandong Province and informed consent was obtained from all patients and their consorts. Data were collected retrospectively from the records of 102 consecutive patients with cervical cancer from May 2007 to January 2009. Participants were within 1-5 years of a cervical cancer diagnosis (FIGO [International Federation of Gynecology and Obstetrics] stage I-III), were currently cancer-free, and had not been diagnosed with another type of cancer. Health-related QoL was measured using the Functional Assessment of Cancer Therapy-Cervical Cancer (FACT-Cx) tool. ${ }^{16}$ We assessed changes in self-reported health status and health-related QoL according to time since diagnosis. The length of cancer survivorship was estimated as time since diagnosis to the date of completing the survey.

\section{Health-related quality of life}

Four subscales from the FACT-Cx were utilized to assess health-related QoL. These were grouped into the following domains: physical well-being, social/family well-being, functional well-being, emotional well-being with six items, and an "additional concerns" domain assessing specific gynecological symptoms, urinary and bowel problems, vaginal changes, and concerns about treatment, diet, sexuality, and self-image. Items are rated from 0 (not at all) to 4 (very much), with a higher score indicating better health-related QoL (Cronbach's alpha coefficient $=0.82$ )

\section{Sexual impact}

An eight-item scale was used to assess the sexual impact of treatment for cervical cancer, and comprised items from previous cancer studies reported by Ashing-Giwa ${ }^{17}$ (three items, ie, sexual desire, pleasure, and relationship) and relevant items from the FACT additional cervical cancer concerns scale (five items). All items are rated using a Likert-type scale. To make a composite score, the item responses were changed to the three-response format, ie, negative, neutral, and positive impact. The eight items were then averaged, with high scores indicating less severe sexual impact. ${ }^{17}$

\section{Health care satisfaction: patient-doctor relationship}

The 38-item Adherence Determinants Questionnaire (ADQ) measures interpersonal aspects of patient care, communication, benefits/costs, severity of illness, adherence, and medical support. ${ }^{18}$ The patient-doctor relationship was assessed using this instrument, and scores on these items were averaged, with higher scores indicating better relationships.

\section{Statistical analysis}

The data were stored and analyzed using SPSS version 17.0 software (SPSS Inc., Chicago, IL, USA). The Cronbach's alpha coefficient was used to test the internal consistency of the items and domains of the FACT-Cx. The reference value adopted as being acceptable was $\geq 0.60 .{ }^{19}$ One-way analysis of variance was used to evaluate the relationship between health-related QoL and patient characteristics. The Pearson product-moment correlation coefficient was used to evaluate the correlation between health-related QoL and the patient-doctor relationship/sexual impact. $P<0.05$ was considered to be statistically significant for all analyses.

\section{Results \\ Sample characteristics}

The median age of the 102 patients at the time of diagnosis was 43.2 (range 23-70 years; 50 women were aged $\geq 50$ years, and 52 women were aged $<50$ years). Four $(3.9 \%)$ patients were diagnosed in FIGO stage I, 59 (57.8\%) were diagnosed in FIGO stage II, and 39 (38.2\%) were diagnosed in FIGO stage III. All patients had squamous cell carcinoma. Eighty-nine were married (87.3\%) and 13 (12.7\%) were widowed/divorced. Annual household income was low in 17 patients, medium in 50, and high in 35 . Of the 102 patients analyzed, the educational levels were illiteracy in 20 patients $(19.6 \%)$, primary in 41 patients $(40.2 \%)$, and secondary or higher in 41 patients $(40.2 \%)$. Fourteen patients received simple radiation therapy and $83(81.4 \%)$ received concurrent chemotherapy (of whom 20 received surgery prior to radiotherapy). Five patients $(4.9 \%)$ received surgery and radiotherapy. Patient characteristics are outlined in Table 1.

\section{Health-related QoL}

As noted in Table 1, mean health-related QoL was 73.99, as measured by the SF-36, in the 102 cervical cancer survivors interviewed. The results showed significant mediating effects 
Table I Relationship between health-related quality of life and patient characteristics

\begin{tabular}{|c|c|c|c|}
\hline & $\mathbf{n}$ & FACT-Cx, mean (SD) & $P$-value \\
\hline \multicolumn{4}{|l|}{ Education } \\
\hline Illiterate & 20 & 70.34 (56.2-78.05) & 0.000 \\
\hline Primary & 41 & $72.02(60.5-77.5)$ & \\
\hline Secondary/higher & 41 & $77.74(66.52-85.0)$ & \\
\hline \multicolumn{4}{|l|}{ FIGO stage } \\
\hline I & 4 & $81.7(79.2-85.0)$ & 0.000 \\
\hline II & 59 & $75.5(71.5-83.25)$ & \\
\hline III & 39 & 70.9I (56.2-73.3) & \\
\hline \multicolumn{4}{|l|}{ Marital status } \\
\hline Married & 89 & $74.80(63.3-85.0)$ & 0.000 \\
\hline Widowed/divorced & 13 & $68.42(56.2-77.5)$ & \\
\hline \multicolumn{4}{|l|}{ Income } \\
\hline Low & 17 & $68.82(56.2-73.5)$ & 0.000 \\
\hline Medium & 50 & $73.22(68.3-77.5)$ & \\
\hline High & 35 & $77.60(73.8-85.0)$ & \\
\hline \multicolumn{4}{|l|}{ Age, years } \\
\hline$\geq 50$ & 50 & $72.54(56.2-75.6)$ & 0.006 \\
\hline$<50$ & 52 & $75.39(74.5-85.0)$ & \\
\hline \multicolumn{4}{|l|}{ Therapy } \\
\hline$S+R+C$ & 20 & $75.25(56.2-85.0)$ & 0.247 \\
\hline $\mathrm{R}+\mathrm{C}$ & 63 & $68.80(63.3-71.5)$ & \\
\hline$S+R$ & 5 & $77.45(75.7-85.0)$ & \\
\hline $\mathrm{R}$ & 14 & $73.58(70.5-82.8)$ & \\
\hline
\end{tabular}

Abbreviations: C, chemotherapy; R, radiotherapy; S, surgery; FACT-Cx, Functional Assessment of Cancer Therapy-Cervical Cancer; FIGO, Internationa Federation of Gynecology and Obstetrics; SD, standard deviation.

of general health status and psychological well-being between life burden and health-related QoL, between sexual impact and health-related QoL, and between the patient-doctor relationship and health-related QoL. In addition, there were a statistically significant relationship between health-related QoL, education level, tumor stage, marital status, and age $(P<0.01)$. However, no significant difference in healthrelated QoL was observed according to which type of treatment the patients had received $(P=0.247)$. In multivariate analysis, sexual functioning was independently correlated with health-related QoL $(P=0.000)$.

\section{Sexual function}

The mean sexual impact score was 2.04. Seventeen patients (16.7\%) were not sexually active at the time of interview, and cited several reasons for no sexual activity (participants were allowed to cite multiple reasons): no partner (13, 12.7\%); not interested and/or had a partner who was not interested $(25,24.5 \%)$; fatigue $(31,30.4 \%)$; physical problems $(30,29.5 \%)$; and other reasons (three, $2.9 \%)$. Sexual functioning was significantly correlated with education level, tumor stage, marital status, income, and age $(P<0.01)$. Univariate analysis indicates that patients with a poor patient- doctor relationship also had problems with sexual function $(P<0.01$, Table 2$)$.

\section{Patient-doctor relationship}

The mean score for the patient-doctor relationship was 3.93. Excluding age and treatment, this relationship was related to other sample characteristics, such as income, education level, tumor stage, and marital status $(P<0.01$, Table 2$)$.

\section{Discussion}

Cervical cancer is one of the more easily detected and highly treatable cancers. The goals of cancer treatment are to cure the disease, prolong survival, and maintain QoL as far as possible. The primary cancer preventive strategies are early diagnosis and timely appropriate care. However, in spite of improved survival rates, the physical and psychosocial effects of treatment remain. Surgery, organ loss, and scar creation have a negative impact on psychophysical identity, causing anxiety and disruption of body image and sexual function. Long-term side effects of chemotherapy and radiotherapy are multiple and well documented, and include loss of ovarian

Table 2 Relationship between sexual impact, patient-doctor relationship, and other patient characteristics

\begin{tabular}{|c|c|c|c|c|}
\hline & $\begin{array}{l}\text { Sexual impact, } \\
\text { mean (SD) }\end{array}$ & $P$-value & $\begin{array}{l}\text { Patient-doctor } \\
\text { relationship, } \\
\text { mean (SD) }\end{array}$ & $P$-value \\
\hline \multicolumn{5}{|l|}{ Education } \\
\hline Illiterate & $1.92(1.68-2.12)$ & 0.000 & 3.71 (3.65-3.89) & 0.000 \\
\hline Primary & $2.01(1.75-2.15)$ & & $3.89(3.83-4.05)$ & \\
\hline $\begin{array}{l}\text { Secondary/ } \\
\text { higher }\end{array}$ & $2.14(1.80-2.25)$ & & $4.07(3.95-4.28)$ & \\
\hline \multicolumn{5}{|l|}{ FIGO stage } \\
\hline I & $2.24(1.86-2.25)$ & 0.000 & $4.23(4.18-4.28)$ & 0.000 \\
\hline II & $2.08(1.73-2.12)$ & & $3.97(3.65-4.20)$ & \\
\hline III & $1.97(1.68-2.03)$ & & $3.84(3.80-3.95)$ & \\
\hline \multicolumn{5}{|l|}{ Marital status } \\
\hline Married & $2.07(1.70-2.25)$ & 0.000 & $3.95(3.65-4.28)$ & 0.000 \\
\hline $\begin{array}{l}\text { Widowed/ } \\
\text { divorced }\end{array}$ & $1.84(1.68-2.05)$ & & $3.75(3.70-3.85)$ & \\
\hline \multicolumn{5}{|l|}{ Income } \\
\hline Low & $\mathrm{I} .90(\mathrm{I} .68-2.0 \mathrm{I})$ & 0.000 & $3.70(3.65-3.78)$ & 0.000 \\
\hline Medium & $2.02(1.89-2.10)$ & & $3.90(3.85-3.93)$ & \\
\hline High & $2.15(2.08-2.25)$ & & $4.08(4.0 \mathrm{I}-4.28)$ & \\
\hline \multicolumn{5}{|l|}{ Age, years } \\
\hline$\geq 50$ & $1.98(1.68-2.05)$ & 0.000 & $3.89(3.65-4.12)$ & 0.052 \\
\hline$<50$ & $2.10(2.05-2.25)$ & & $4.06(3.78-4.28)$ & \\
\hline \multicolumn{5}{|l|}{ Therapy } \\
\hline$S+R+C$ & $2.07(1.79-2.12)$ & 0.494 & $4.00(3.88-4.05)$ & 0.094 \\
\hline$R+C$ & $1.95(1.68-2.23)$ & & $3.91(3.65-4.20)$ & \\
\hline$S+R$ & $2.10(2.01-2.15)$ & & $4.03(3.99-4.28)$ & \\
\hline $\mathrm{R}$ & $2.02(1.98-2.25)$ & & $3.87(3.79-4.15)$ & \\
\hline
\end{tabular}

Abbreviations: $\mathrm{C}$, chemotherapy; R, radiotherapy; S, surgery; FACT-Cx, Functiona Assessment of Cancer Therapy-Cervical Cancer; FIGO, International Federation of Gynecology and Obstetrics; SD, standard deviation. 
function, nausea, vomiting, fatigue, alopecia, depression, skin changes, vaginal atrophy, and diarrhea. ${ }^{20,21}$ Further, the sexual and reproductive side effects resulting from treatment for cervical cancer include reduced sexual desire, premature menopause, loss of fertility, and reduction in vaginal elasticity, often resulting in pain and impaired sexual function. Thus, the health-related QoL measurement in oncology is important.

In general, our study shows that better physical and psychosocial status was significantly associated with less life burden, better sexual function, and a good patient-doctor relationship. Education level, tumor stage, marital status, and age were also related to health-related QoL. The results document even greater physical and psychosocial challenges for cervical cancer survivors with lower incomes. This finding seems to be consistent with results from another study, ${ }^{22}$ suggesting that life burden is one of the key predictors associated with health-related QoL. The significant financial strain influencing health-related QoL was endorsed by cancer survivors. Further, women have the primary responsibility for caring for all members of the household according to traditional gender roles, which determines family dynamics in Chinese culture. Such a role may add a particular burden in daily life for women who are cancer survivors.

The findings reflect persistent concerns regarding the physical and psychosocial domains reported in the literature for cervical cancer survivors. As anticipated, sexual functioning appears to be compromised due to physical discomfort, with survivors reporting a greater incidence of vaginal dryness and hot flashes. In our study, three of the 13 participants who divorced or separated after being diagnosed with cancer indicated that this change was related to their disease, and sexual functioning was independently correlated with their health-related QoL. Further, survivors with a lower income and limited education appeared to experience much greater distress. Women who reported greater survivor-specific distress also reported less confidence managing their illness, more reproductive concerns, worse general health, diminished spirituality and well-being, and less social support. However, our study shows that the treatment modalities used did not affect health-related QoL or sexual functioning, which seems to be in contrast with another report that radiotherapy and chemoradiotherapy is associated with sexual and intestinal dysfunction in women with cervical carcinoma. ${ }^{10}$ Therefore, it is necessary to examine further the contribution that each of these variables make in predicting who is more likely to experience persistent vulnerabilities associated with survivorship.

Moreover, the burden of socioeconomic stress further exacerbates poor health-related QoL outcomes. A good patient-doc- tor relationship has the highest correlation with health-related QoL. Enhanced communication increases understanding, trust, and adherence. Information about cervical cancer and healthrelated QoL needs to be communicated. A good patient-doctor relationship can be an effective medium for increasing awareness and understanding of cervical cancer and its treatment, particularly for women who are illiterate or have had limited schooling. Our survivors reported problems with the health care system that resulted in negative treatment sequelae, some of which could have been avoided. This is consistent with other data suggesting that survivors who report less social support are more likely to utilize multiple coping strategies to manage illness-related stressors. ${ }^{11,23}$ It has been speculated that those with less social support and other psychosocial limitations attempt to compensate by continued active coping. ${ }^{11}$ Those cured of disease should theoretically have little objective evidence of illness. Therefore, the strength of this relationship may be increased for underserved (patients with less social support and other psychosocial limitations) populations.

US-based studies of health-related QoL in cervical cancer survivors have primarily focused on European American women, whereas Latin Americans are overrepresented in the cervical cancer statistics, and experience poorer healthrelated QoL. ${ }^{17}$ Ashing-Giwa et al described the experience of cancer in a diverse sample of cervical cancer survivors, ie, African American, Latin American, Asian American, and Caucasian women from 23 key informant interviews and 51 focus group interviews. They reported moderate health-related QoL in their participants. However, persistent concerns included the side effects of treatment, difficulties accessing quality care, inadequate health insurance, barriers to a good physician-patient relationship such as language and doctors' time constraints, insufficient knowledge about cervical cancer due to limited information from medical staff, along with lack of control over treatment, social support, family well-being, functional, and work issues, and sexuality and relationship concerns. Socioeconomic status, ethnicity, cultural beliefs and practices, age, and family support dictated the level of cancer-related burden. ${ }^{24}$

In 2011, Ashing-Giwa et al showed the significant mediating effects of general health status and psychological well-being between the patient-doctor relationship and health-related QoL, and between life burden and health-related QoL, respectively, for all ethnic and language groups. ${ }^{17}$ Our findings are similar to those of a previous study in the USA ${ }^{17}$ In addition, other studies have shown that time since diagnosis affects self-reported health status and QoL among cancer survivors. $^{25,26}$ Thus, further research would be anticipated 
in this area in the People's Republic of China, although not included as part of our present study.

In summary, this study presents new findings reflecting interconnectedness among the dimensions of health-related QoL. The results also advanced our understanding of predictors of health-related QoL and the relationship between them. However, our study has some limitations arising from its retrospective design and the data being obtained from a single institution. Therefore, more patient charts should be reviewed in the future to obtain more information about health-related QoL in Chinese cervical cancer survivors. Despite these limitations, our findings still highlight the pressing need for more research on health-related QoL in the diverse populations of cervical cancer survivors in the People's Republic of China. Our present results could inform future investigations on health-related QoL, psychosocial burden, and health disparities among cervical cancer survivors.

\section{Conclusion}

The findings of this study advance our understanding of the predictors of health-related QoL and the relationship between these in cervical cancer survivors. Health-related QoL in these women may be improved by alleviating their life burden and sexual function, and by improving the patientdoctor relationship.

\section{Disclosure}

The authors report no competing interests in this work.

\section{References}

1. American Cancer Society. Cancer Facts and Figures. 2005.

2. Sharma R. HPV vaccine: a breakthrough in prevention of cervical cancer. Apollo Medicine. 2012;9:87-90.

3. Gao Y, Gao F, Liu Z, Song LP. Treatment results of incomplete chemoradiotherapy in locally advanced cervical cancer. Onco Targets Ther. 2013;6:297-302.

4. Herrera FG, Prior JO. The role of PET/CT in cervical cancer. Front Oncol. 2013;3:34

5. Gillison ML, Castellsagué X, Chaturvedi A, et al. Comparative epidemiology of HPV infection and associated cancers of the head and neck and cervix. Int J Cancer. 2014;134:497-507.

6. Evans DW, McCartney CF, Nemeroff CB, et al. Depression in women treated for gynecological cancer: clinical and neuroendocrine assessment. Am J Psychiatry. 1986;143:447-452.

7. Vistad I, Fossa SD, Dahl AA. A critical review of patient-rated quality of life studies of long-term survivors of cervical cancer. Gynecol Oncol. 2006;102:563-572.

8. Chase DM, Watanabe T, Monk BJ. Assessment and significance of quality of life in women with gynecologic cancer. Future Oncol. 2010;6: $1279-1287$.
9. Mantegna G, Petrillo M, Fuoco G, et al. Long-term prospective longitudinal evaluation of emotional distress and quality of life in cervical cancer patients who remained disease-free 2-years from diagnosis. BMC Cancer. 2013;13:127.

10. de Noronha AF, de Figueiredo EM, Franco TM, Cândido EB, SilvaFilho AL. Treatments for invasive carcinoma of the cervix: what are their impacts on the pelvic floor functions? Int Braz J Urol. 2013;39: $46-54$.

11. Le Borgne G, Mercier M, Woronoff AS, et al. Quality of life in longterm cervical cancer survivors: a population-based study. Gynecol Oncol. 2013;129:222-228.

12. Shaverdian N, Gondi V, Sklenar KL, et al. Effects of treatment duration during concomitant chemoradiation therapy for cervical cancer. Int $J$ Radiat Oncol Biol Phys. 2013;86:562-568.

13. Frumovitz M, Sun CC, Schover LR, et al. Quality of life and sexual functioning in cervical cancer survivors. J Clin Oncol. 2005;23: 7428-7436.

14. Bergmark K, Avall-Lundqvist E, Dickman PW, Henningsohn L, Steineck G. Vaginal changes and sexuality in women with a history of cervical cancer. N Engl J Med. 1999;340:1383-1389.

15. Belhadj H, Rasanathan JJ, Denny L, Broutet N. Sexual and reproductive health and HIV services: integrating HIV/AIDS and cervical cancer prevention and control. Int J Gynaecol Obstet. 2013;121 Suppl 1: S29-S34.

16. Cella DF, Tulsky DS, Gray G, et al. The Functional Assessment of Cancer Therapy scale: development and validation of the general measure. J Clin Oncol. 1993;11:570-579.

17. Ashing-Giwa KT, Lim JW. Health-related quality of life outcomes among cervical cancer survivors: examining ethnic and linguistic differences. Cancer Epidemiol. 2011;35:194-201.

18. DiMatteo M, Hays R, Gritz E, Bastani R. Patient adherence to cancer control regimens: scale development and initial validation. Psychol Assess. 1993;5:102-112.

19. Hair JF, Anderson RE, Tatham RL, Black WC. [Multivariate analysis]. Analise Multivariada de Dados. Porto Alegre, Brazil: Bookman. 2005:89-127.

20. Jones GL, Ledger W, Bonnett TJ, Radley S, Parkinson N, Kennedy SH. The impact of treatment for gynecological cancer on health-related quality of life (HRQoL): a systematic review. Am J Obstet Gynecol. 2006;194:26-42.

21. Ferrandina G, Margariti PA, Smaniotto D, et al. Long-term analysis of clinical outcome and complications in locally advanced cervical cancer patients administered concomitant chemoradiation followed by radical surgery. Gynecol Oncol. 2010;119:404-410.

22. Ell K, Xie B, Wells A, Nedjat-Haiem F, Lee PJ, Vourlekis B. Economic stress among low-income women with cancer: effects on quality of life. Cancer. 2008;112:616-625.

23. Wenzel LB, Fairclough DL, Brady MJ, et al. Age-related differences in the quality of life of breast carcinoma patients after treatment. Cancer. 1999;86:1768-1774.

24. Ashing-Giwa KT, Kagawa-Singer M, Padilla GV, et al. The impact of cervical cancer and dysplasia: a qualitative, multiethnic study. Psychooncology. 2004;13:709-728.

25. Schag CA, Ganz PA, Wing DS, Sim MS, Lee JJ. Quality of life in adult survivors of lung, colon, and prostate cancer. Qual Life Res. 1994;3: $127-141$.

26. Fairley TL, Hawk H, Pierre S. Health behaviors and quality of life of cancer survivors in Massachusetts, 2006: data use for comprehensive cancer control. Prev Chronic Dis. 2010;7:A09. 


\section{Publish your work in this journal}

OncoTargets and Therapy is an international, peer-reviewed, open access journal focusing on the pathological basis of all cancers, potential targets for therapy and treatment protocols employed to improve the management of cancer patients. The journal also focuses on the impact of management programs and new therapeutic agents and protocols on

patient perspectives such as quality of life, adherence and satisfaction. The manuscript management system is completely online and includes a very quick and fair peer-review system, which is all easy to use. Visit http://www.dovepress.com/testimonials.php to read real quotes from published authors.

Submit your manuscript here: http://www.dovepress.com/oncotargets-and-therapy-journal 